\title{
Building a European Data Economy
}

\author{
Herbert Zech
}

Published online: 28 June 2017

(C) Max Planck Institute for Innovation and Competition, Munich 2017

On 10 January 2017, the European Commission issued a Communication concerning the building of a European data economy. The Communication is revolutionary in several aspects. For the first time a legislative body addresses questions of data ownership and data access.

Public data have already been discussed and regulated for quite a while, especially with the Directive on the re-use of public sector information. With the Communication, the impact of big data and "Industry 4.0" (cyber-physical systems in industrial production) has finally led to new legislative concepts concerning private data. Previously, this had only been debated within the realm of data protection. Whether personal data could be used as contractual consideration for services is one of the topics that have already been addressed by the European Commission in the proposal for a Directive on certain aspects concerning contracts for the supply of digital content. The recent Communication focuses on machinegenerated non-personal data defined as "data created without the direct intervention of a human by computer processes, applications or services, or by sensors processing information received from equipment, software or machinery, whether virtual or real".

The question "who owns the data?" which used to be a merely academic topic is answered in a novel way. As one of several "possibilities for addressing the issue of access to machine-generated data" the Communication proposes a "data producer's right". Such a right would encompass the use of non-personal raw data, especially for big data analyses. The transferable right would originally be granted to the data producer defined as the "owner or long-term user of a device". In an accompanying questionnaire data producers are also defined as "persons or entities that operate sensor-equipped machines, tools or devices at their own economic risk". The

\section{H. Zech (ه)}

Dr. iur.; Professor of Life Sciences Law and Intellectual Property Law, University of Basel, Basel, Switzerland e-mail: herbert.zech@unibas.ch 
underlying rationale - to allocate benefits and risks of operating smart devices in parallel - is underlined by the fact that the Communication also addresses liability issues. It should be noted that many commentators are concerned about the introduction of such a new right, especially due to possible undesired economic effects. Nevertheless, the proposed right clearly gives new impulses to the area of IP rights. A promising alternative is the introduction of a non-exclusive property right which could also be attributed to the data producer.

Default contract rules are explicitly mentioned as another (ideally accompanying) measure. Such rules could provide a "benchmark balanced solution for contracts relating to data". Moreover, the Communication considers a broadening of the existing unfair terms control by introducing an unfairness control in business-tobusiness contracts. This approach, being the object of hot debates, could create the level playing field and the legal certainty demanded by the German industry.

Almost revolutionary, the Communication also proposes access rights against remuneration. Currently only available as a competition law remedy (and only discussed in theory) such access rights might be granted on a FRAND basis (modelled on competition law) and introduced as a horizontal or as sector-specific measures. Where data monopolies lead to inefficient data use, such rights might be a powerful instrument. Access rights can (and should) be combined with other proposed measures like the data producer's right. Most likely, sector-specific rules will be the way forward, e.g. for connected cars and automated driving.

The Communication addresses even more ideas about data and data technology. Beside measures against localization restrictions, technical guidelines and access for public bodies, very remarkably, liability questions are also discussed. The Commission obviously sees a clear connection between data access and liability (or benefit and risk allocation, as already mentioned). Emerging technologies such as the Internet of Things and highly automated systems (especially self-driving vehicles which arguably will be the first large scale "test case") might require novel liability rules. Although it is not the data use which creates risks for body or property (but, possibly, for privacy), it is the data-generating machine that does. Emerging information technology will create new risks. Connectivity will probably lead to problems in determining causality and responsibility. The Communication rightly states that the provision of certainty to both users and manufacturers is of central importance.

It will be interesting to see how the future discussion about machine-generated data relates to data protection. From an academic perspective there is no reason to restrict the proposed measures to non-personal data. However, the resulting overlaps of different legal regimes might be overly complicated from a practical perspective. The interplay between data protection and legal rules for machine-generated data will be an important issue for further discussion. An interesting concept that bridges the two underlying concepts is the recently proposed right to non-data collecting products. It might help with creating a fair balance between data privacy and data economy.

All in all, the Communication is a remarkable paper. It proposes several novel ideas for data regulation and will also influence the academic discussion. With its extensive accompanying staff working document, it also provides valuable 
information on the current debate. Europe clearly drives the discussion about data governance. 